\title{
A symplectic method for rigid-body molecular simulation
}

\author{
Ayla Kol ${ }^{\mathrm{a})}$ and Brian B. Laird ${ }^{\mathrm{b})}$ \\ Department of Chemistry, University of Kansas, Lawrence, Kansas 66045 \\ Benedict J. Leimkuhler ${ }^{\mathrm{c})}$ \\ Department of Mathematics, University of Kansas, Lawrence, Kansas 66045
}

(Received 20 March 1997; accepted 12 May 1997)

\begin{abstract}
Rigid-body molecular dynamics simulations typically are performed in a quaternion representation. The nonseparable form of the Hamiltonian in quaternions prevents the use of a standard leapfrog (Verlet) integrator, so nonsymplectic Runge-Kutta, multistep, or extrapolation methods are generally used. This is unfortunate since symplectic methods like Verlet exhibit superior energy conservation in long-time integrations. In this article, we describe an alternative method, which we call RSHAKE (for rotation-SHAKE), in which the entire rotation matrix is evolved (using the scheme of McLachlan and Scovel [J. Nonlin. Sci. 16233 (1995)]) in tandem with the particle positions. We employ a fast approximate Newton solver to preserve the orthogonality of the rotation matrix. We test our method on a system of soft-sphere dipoles and compare with quaternion evolution using a 4th-order predictor-corrector integrator. Although the short-time error of the quaternion algorithm is smaller for fixed time step than that for RSHAKE, the quaternion scheme exhibits an energy drift which is not observed in simulations with RSHAKE, hence a fixed energy tolerance can be achieved by using a larger time step. The superiority of RSHAKE increases with system size. (C) 1997 American Institute of Physics. [S0021-9606(97)50931-7]
\end{abstract}

\section{INTRODUCTION}

An important problem in molecular simulation is the development of stable and efficient algorithms for integrating the equations of motion of orientational degrees of freedom. The straightforward parameterization for such degrees of freedom, Euler angles, ${ }^{1}$ is at a severe disadvantage for numerical work because of the singularities inherent in the description. To overcome this difficulty, a parameterization based on quaternions was proposed by Evans ${ }^{2}$ and has become standard. ${ }^{3}$ A significant drawback in this approach arises from the fact that the rigid-body Hamiltonian is nonseparable in the quaternion representation, therefore preventing the use of the popular leapfrog (Verlet) algorithm for integrating the rotational equations of motion. [In this article, we follow the convention of the recent literature on symplectic integration ${ }^{4}$ and say that a Hamiltonian is separable if it can be written in the form $H=T(p)+V(q)$.] Usually, then, higher-order methods such as Gear predictor-corrector methods are utilized. The superiority of the Verlet scheme arises from the fact that it is, like the true Hamiltonian dynamics, symplectic; that is, it preserves the wedge product $d r \wedge d p$ ( $p$ is the momentum conjugate to $r)^{4,5}$ A more familiar, but weaker property of symplectic maps, is that the flow preserves volumes in phase space. Such symplectic integration algorithms have been shown to possess excellent long-term energy stability, often far superior to nonsymplectic methods (even those with higher-order local error). For a

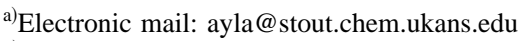

${ }^{b)}$ Electronic mail: laird@pilsner.chem.ukans.edu

${ }^{c}$ Electronic mail: leimkuhl@math.ukans.edu
}

recent example showing the improved long-term stability of integration methods incorporating geometric structure, see Ref. 6.

For systems of rigid molecules in which the potential is easily written in terms of intermolecular interactions between atomic sites on the molecules, it is possible to circumvent the parameterization of orientational degrees of freedom by considering the fundamental variables to be the individual Cartesian coordinates of the atomic sites. The dynamics is determined by integrating the equations of motion for these sites, subject to the constraint that the molecule remains rigid (intramolecular bond distances and angles are fixed). The constraints can be implemented using appropriate Lagrange multipliers. A generalization of the Verlet integration scheme (SHAKE algorithm ${ }^{7}$ ) is then used to integrate the constrained equations of motion. SHAKE is algebraically equivalent to the RATTLE discretization of Anderson, ${ }^{8}$ and the latter scheme is formally symplectic along the manifold of constraints. ${ }^{9}$ This fact helps to explain the excellent longtime stability of SHAKE. The use of SHAKE on a constrained particle description becomes inconvenient for general rigid-body integration for two reasons. First, as the number of atoms in each rigid molecule increases, the number of constraints increases dramatically, which decreases the efficiency of the computation. Second, additional computational complexity is introduced in the force computations for intermolecular potentials that are not easily decomposable into direct site-site interactions such as potentials expressed as multipole expansions.

In this paper, we develop an algorithm in which the rotation matrix is not parameterized, but is evolved directly. The structure of the equations of motion is such that a SHAKE/RATTLE scheme is possible, except that, instead of 
constraining the bond lengths (or angles), we constrain the rotation matrix to be orthogonal. ${ }^{10,11} \mathrm{We}$ also describe efficient iteration procedures for the nonlinear equations that must be solved at each time step. We will refer to this approach as RSHAKE (for rotation SHAKE). The advantages of this model over SHAKE with bond constraints are that the number of constraints does not increase with molecular size, and that it is well suited for non-site-to-site interactions such as those generated from multipole expansions.

\section{SIMULATION OF RIGID-BODY MOTION}

We consider a collection of $N$ interacting rigid bodies. The time evolution of a rigid body can be studied by considering separately the translational motion of the center of mass and the rotations about the principal axes (which pass through the center of mass). The Lagrangian for such a system is

$$
\mathscr{C}=\sum_{i} \frac{\mathbf{v}_{i}^{T} M_{i} \mathbf{v}_{i}}{2}+\sum_{i} \frac{\boldsymbol{\Omega}_{i}^{T} I_{i} \mathbf{\Omega}_{i}}{2}-\Phi\left(\left\{\mathbf{r}_{i}\right\},\left\{\theta_{i}\right\}\right),
$$

where $\mathbf{r}_{i}, \mathbf{v}_{i}, \boldsymbol{\Omega}_{i}$, and $I_{i}$ are the Cartesian position vector of the center of mass, its velocity, the (body-frame) angular velocity, and the moment-of-inertia tensor (also in the body frame) of particle $i$, respectively. $\Phi$ is the potential energy function and $\theta_{i}$ is a representation of the orientation of particle $i$. The equations for center-of-mass motion are

$$
\begin{aligned}
& \dot{\mathbf{r}}_{i}=\mathbf{v}_{i}, \\
& M_{i} \dot{\mathbf{v}}_{i}=-\nabla_{\mathbf{r}_{i}} \Phi .
\end{aligned}
$$

Numerically, these can be integrated effectively by using the leapfrog (Verlet) method:

$$
\begin{aligned}
& \mathbf{r}_{n+1, i}=\mathbf{r}_{n, i}+\Delta t \mathbf{v}_{n+1 / 2, i}, \\
& M_{i} \mathbf{v}_{n+1 / 2, i}=M_{i} \mathbf{v}_{n-1 / 2, i}-\Delta t \nabla_{\mathbf{r}_{i}} \Phi\left(\mathbf{r}_{n, i}\right) .
\end{aligned}
$$

(Here the notation $\mathbf{r}_{n, i}$ refers to the $i$ th position variable at the $n$th time step.)

To determine the rotational motion, one needs to make use of two different reference frames: a space-fixed frame (also called the laboratory-fixed frame) and a body-fixed frame. Forces and torques are more conveniently calculated in the laboratory frame whereas the rotational equations of motion are simpler in the body frame. We will use the convention that small letters stand for the representations of variables in the fixed laboratory frame and their counterparts in the body frame will be denoted by capital letters. With this convention we have

$$
\mathbf{r}=Q \mathbf{R},
$$

where $\mathbf{r}$ and $\mathbf{R}$ are vectors in the laboratory and body frames, respectively, and $Q$ is the $3 \times 3$ time-dependent rotation matrix subject to the orthogonality condition

$$
Q Q^{T}=1=Q^{T} Q
$$

A consequence of this condition is that only three independent parameters are necessary to describe the nine elements of the rotation matrix.

One standard parametrization of $Q$ is in terms of the well-known Euler angles, ${ }^{1}$ but the singularities inherent in this description make it unsuitable for numerical work. Another is the quaternion parametrization of Hamilton, where the orthogonality condition is again explicitly implemented utilizing four quaternions instead of the three Euler angles. The quaternion method and its implementation is outlined in the next section.

In Sec. IV we present a rotational evolution algorithm for molecular simulations (RSHAKE) that is both explicit and algebraically equivalent to a symplectic method. The method is compared in Sec. VI for a system of dipolar soft spheres (described in Sec. V) with a quaternion method using a fourth-order (Gear) predictor corrector integrator.

\section{QUATERNION METHOD}

In the quaternion representation, ${ }^{2}$ the orientation of a rigid body is parameterized in terms of a set of four scalar quantities, $\left(q_{0}, q_{1}, q_{2}, q_{3}\right) \equiv \mathbf{q}$, with the condition

$$
q_{0}^{2}+q_{1}^{2}+q_{2}^{2}+q_{3}^{2}=1
$$

In terms of the standard Euler angles, ${ }^{1,3} \theta, \phi$, and $\psi$, the quaternion parameters are

$$
\begin{aligned}
& q_{0}=\cos \left(\frac{\theta}{2}\right) \cos \left(\frac{\phi+\psi}{2}\right), \\
& q_{1}=\sin \left(\frac{\theta}{2}\right) \cos \left(\frac{\phi-\psi}{2}\right), \\
& q_{2}=\sin \left(\frac{\theta}{2}\right) \sin \left(\frac{\phi-\psi}{2}\right), \\
& q_{3}=\cos \left(\frac{\theta}{2}\right) \sin \left(\frac{\phi+\psi}{2}\right),
\end{aligned}
$$

and the rotation matrix is given by

$$
Q=\left(\begin{array}{lll}
q_{0}^{2}+q_{1}^{2}-q_{2}^{2}-q_{3}^{2} & 2\left(q_{1} q_{2}-q_{0} q_{3}\right) & 2\left(q_{1} q_{3}+q_{0} q_{2}\right) \\
2\left(q_{1} q_{2}+q_{0} q_{3}\right) & q_{0}^{2}-q_{1}^{2}+q_{2}^{2}-q_{3}^{2} & 2\left(q_{2} q_{3}-q_{0} q_{1}\right) \\
2\left(q_{1} q_{3}-q_{0} q_{2}\right) & 2\left(q_{2} q_{3}+q_{0} q_{1}\right) & q_{0}^{2}-q_{1}^{2}-q_{2}^{2}+q_{3}^{2}
\end{array}\right)
$$


where the normalization condition [Eq. (6)] ensures the orthogonality of $Q$. The introduction of these four quaternion parameters (plus a constraint) in place of the three Euler angles removes the singularities of the Euler angle representation. ${ }^{1}$

Then in the quaternion representation, the equations of motion for the angular velocities augmented with the time derivative of the constraint [Eq. (6)] on the quaternions results in

$$
\frac{d \mathbf{q}}{d t}=\mathscr{Q} \mathbf{\Omega},
$$

where

$$
\mathscr{b}=\frac{1}{2}\left(\begin{array}{cccc}
q_{0} & -q_{1} & -q_{2} & -q_{3} \\
q_{1} & q_{0} & -q_{3} & q_{2} \\
q_{2} & q_{3} & q_{0} & -q_{1} \\
q_{3} & -q_{2} & q_{1} & q_{0}
\end{array}\right)
$$

and

$$
\boldsymbol{\Omega}=\left(\begin{array}{c}
0 \\
\Omega_{x} \\
\Omega_{y} \\
\Omega_{z}
\end{array}\right)
$$

is the angular velocity in body frame. The time derivative of the angular velocity is related to the torque $\mathscr{T}$ and the principal moments of inertia in the body frame by

$$
\begin{gathered}
\dot{\Omega}_{x}=\frac{\mathscr{T}_{x}}{I_{x x}}+\left(\frac{I_{y y}-I_{z z}}{I_{x x}}\right) \Omega_{y} \Omega_{z}, \\
\dot{\Omega}_{y}=\frac{\mathscr{T}_{y}}{I_{y y}}+\left(\frac{I_{z z}-I_{x x}}{I_{y y}}\right) \Omega_{z} \Omega_{x}, \\
\dot{\Omega}_{z}=\frac{\mathscr{T}_{z}}{I_{z z}}+\left(\frac{I_{x x}-I_{y y}}{I_{z z}}\right) \Omega_{x} \Omega_{y} .
\end{gathered}
$$

The above seven coupled equations can be integrated numerically using a variety of methods. The fact that the time derivatives of the quaternions depend not only on the angular velocity $\boldsymbol{\Omega}$, but also on the quaternions themselves through $\mathscr{b}$, means that the standard leapfrog (Verlet) algorithm is not applicable. For this reason, higher-order nonsymplectic algorithms such as Bulirsch-Stoer extrapolation, standard Runge-Kutta methods, or the Gear predictorcorrector method (see Appendix E of Ref. 3) are typically employed.

Recently Fincham ${ }^{12}$ has devised an implicit leapfrog"like" integration algorithm for quaternions. His method is based on Eq. (9) and the equation of motion for the angular momentum

$$
\frac{d \mathbf{l}}{d t}=\boldsymbol{\tau},
$$

where $l$ is the three-dimensional angular momentum vector in the laboratory frame and $\tau$ is the torque in the same coordinate system. The implicit algorithm of Fincham then proceeds as follows

$$
\mathbf{l}_{n+1 / 2}=\mathbf{l}_{n-1 / 2}+\Delta t \boldsymbol{\tau}_{n},
$$

$$
\mathbf{q}_{n+1}=\mathbf{q}_{n}+\frac{\Delta t}{2}\left(\mathscr{C}_{n} \mathbf{\Omega}_{n}+\mathscr{C}_{n+1} \boldsymbol{\Omega}_{n+1}\right) .
$$

The last equation is an implicit one defined through the auxiliary parameters $\mathbf{l}_{n}=\mathbf{l}_{n-1 / 2}+\Delta t \boldsymbol{\tau}_{n}$ and $\mathbf{l}_{n+1}=\mathbf{l}_{n}+\Delta t \boldsymbol{\tau}_{n}$, which are not stored, but used to calculate the angular velocities in the body frame

$$
\boldsymbol{\Omega}_{n}=\left(\begin{array}{c}
0 \\
I^{-1} C_{n}^{T} \mathbf{l}_{n}
\end{array}\right) .
$$

In our implementation of this method, discussed in Sec. VI, this method of Fincham, while comparable in per-step efficiency to the 4th-order predictor-corrector algorithm for quaternions, exhibited much worse stability than the alternatives considered.

\section{THE RSHAKE METHOD}

In RSHAKE, the rotation matrix $\mathcal{Q}$ is evolved directly, as opposed to evolving a set of parameters (Euler angles or quaternions) used to represent $\mathcal{Q}$. In doing so, we use a scheme similar to that of McLachlan and Scovel ${ }^{10}$ (see also Reich ${ }^{11}$ ), in which one defines a momentum variable canonically conjugate to $Q$ - the resulting Hamilton's equations of motion subject to the orthogonality constraint $Q^{T} Q=1$ are then of the proper form for the implementation of efficient symplectic integrators such as SHAKE/RATTLE.

A basic property of Hamiltonian systems is that the flow (time evolution of coordinates and momenta) is symplectic, which for mechanical systems with one degree of freedom, such as a simple harmonic oscillator, means that the area in two-dimensional phase space is invariant during time evolution. (For systems with more than one degree of freedom, the conserved quantity is the sum of the projected areas, onto each $\left(p_{i}, q_{i}\right)$ plane, of any arbitrary oriented twodimensional surface in phase space.) In general, symplecticness implies volume preservation in phase space (the Liouville theorem), but it is a stronger condition. It has been found to be very desirable to maintain the symplectic invariance during numerical integration. For an overview, the reader is referred to Ref. 7.

The concept of a symplectic numerical method can be extended to constrained dynamics ${ }^{9,13}$ compactly described by $H=T(p)+V(q)+g(q) \cdot \lambda, g(q)=0$. The SHAKE discretization $^{7}$ generalizes the leapfrog method to constrained systems, and the symplecticness of the leapfrog method carries over to SHAKE. A rigorous treatment of this fact and generalization of this argument to constrained mechanical systems can be found in Ref. 9, for both SHAKE and the closely related RATTLE method. ${ }^{8}$

Although it is possible to formulate the quaternions as a Hamiltonian system, ${ }^{14}$ the equations then are in nonseparable form, and the available symplectic methods for such problems are implicit, meaning that the symplectic structure is maintained only if a certain nonlinear system of equations involving all the force contributions is solved exactly at each time step. This is generally expensive. Instead, we make use of an alternative formulation, writing the equations of motion 
in the form of a separable constrained Hamiltonian system for the rotation matrix $\mathcal{Q}$. This approach also requires the solution of a system of nonlinear equations at each time step, but, critically, only one evaluation of the interbody forces will be needed at each step.

The rotational kinetic energy must be expressed in terms of the time derivative of $\mathscr{Q}$. This is done by considering the molecule to be made up of discrete atoms of mass $m_{j}$ centered at position $\mathbf{R}_{j}$ in the body-fixed frame. (The total mass of the molecule is then $M=\Sigma_{j} m_{j}$.) The rotational kinetic energy of the molecule, $K_{\text {rot }}$, is then given by

$$
K_{\mathrm{rot}}=\frac{1}{2} \sum_{i} m_{i} \mathbf{v}_{i} \cdot \mathbf{v}_{i}=\frac{1}{2} \sum_{i} m_{i} \operatorname{Tr}\left[\dot{\mathscr{Q}} \mathbf{R}_{i} \mathbf{R}_{i} \dot{\mathscr{Q}}^{T}\right]
$$

since $\mathbf{v}_{i}=\dot{Q} \mathbf{R}_{i}$. Defining a body-frame tensor

$$
J \equiv \sum_{i} m_{i} \mathbf{R}_{i} \mathbf{R}_{i},
$$

gives

$$
K_{\text {rot }}=\frac{1}{2} \operatorname{Tr}\left[\dot{Q} J \dot{Q}^{T}\right] .
$$

For a continuous mass distribution described by a mass density $\rho(\mathbf{R})$, we have

$$
J \equiv \int d \mathbf{R} \rho(\mathbf{R}) \mathbf{R} \mathbf{R} .
$$

Note that $J$ differs slightly from the moment-of-inertia tensor $I$, so that if, as usual, the body frame is chosen to be such that $J$ (and therefore $I$ ) is diagonal, we have

$$
I_{\alpha \alpha}=J_{\beta \beta}+J_{\gamma \gamma}
$$

where $(\alpha, \beta, \gamma)$ is a cyclic permutation of $(1,2,3)$.

In terms of $\mathcal{Q}$, the Lagrangian for a single rigid body with center-of-mass position vector $\mathbf{r}_{\mathrm{cm}}$ is then

$$
\begin{aligned}
\mathscr{C}= & \frac{M}{2} \dot{\mathbf{r}}_{\text {c.m. }} \cdot \dot{\mathbf{r}}_{\text {c.m. }}+\frac{1}{2} \operatorname{Tr}\left[\dot{Q} \mathrm{~J} \dot{\mathscr{Q}}^{T}\right]-\Phi\left(Q, \mathbf{r}_{\text {c.m. }}\right) \\
& +\operatorname{Tr}\left[\lambda\left(Q^{T} Q-1\right)\right] .
\end{aligned}
$$

The first and the third terms are the usual translational kinetic energy and the total potential energy. The last term, involving the Lagrange multiplier matrix $\lambda$, has been added to ensure that the equations of motion are such that the rotation matrix remains orthogonal. $\lambda$ is a symmetric matrix since it enforces a constraint on a matrix which is symmetric.

To construct the rotational equations of motion, we define the momentum $\Pi$ conjugate to $\mathscr{Q}$ in the usual way

$$
\Pi \equiv \frac{\partial \mathscr{C}}{\partial \dot{\mathscr{Q}}}=\dot{Q} J
$$

(Like $Q, \Pi$ is a $3 \times 3$ matrix.) The rotational kinetic energy becomes

$$
K_{\text {rot }}=\frac{1}{2} \operatorname{Tr}\left[\Pi J^{-1} \Pi^{T}\right] .
$$

Hamilton's equations for the rotational motion are then

$$
\dot{Q}=\Pi J^{-1} \text {, }
$$

$$
\begin{aligned}
& \dot{\Pi}=-\partial_{Q} \Phi+2 Q \lambda, \\
& g(Q)=Q^{T} Q-1=0 .
\end{aligned}
$$

The structure of these equations is such that a SHAKE/ RATTLE integration algorithm is applicable:

$$
\begin{aligned}
& \Pi_{n+1 / 2}=\Pi_{n-1 / 2}+\Delta t\left[2 Q_{n} \lambda_{n}-\partial_{Q_{n}} \Phi\right], \\
& Q_{n+1}=Q_{n}+\Delta t \Pi_{n+1 / 2} J^{-1}, \\
& g\left(Q_{n+1}\right)=0 .
\end{aligned}
$$

Equations (28)-(30) define RSHAKE (for rotational SHAKE). We now have $2 \times 9=18$ parameters to evolve instead of the 7 for quaternions, plus the added work to solve for the 6 independent elements of $\lambda$. A discussion of a variety of methods for solving the nonlinear constraint equations is included in Appendix A. If the constraint is iterated to convergence, the local error occurring after one step with the RSHAKE integrator is $O\left((\Delta t)^{3}\right)$. (Note that, for planar and linear molecules the matrix $J$ is not invertible. For these cases, minor modifications in the equations of motion and the RSHAKE algorithm are required. These are discussed in Appendix B.)

It should be noted that a method for propagating the rotation matrix directly was presented by Ahlrichs and Brode $^{15}$ - the relation to the present work is not immediate, since the notations differ substantially. The method of Ahlrichs and Brode does not, as in RSHAKE, solve the constraint equation to within a small multiple of the computer's unit roundoff error, but instead writes the rotation matrix as an exponential of some antisymmetric matrix $A$ and, using information about $A$ from the last step, determines the new value of $A$ to third order in $\Delta t$ by expanding the equations of motion. The new $A$ is then exponentiated to give the new rotation matrix-which is guaranteed to be orthogonal due to the antisymmetry property of $A$. From the point of view of the present paper, the most important drawback of their procedure is that it will not be symplectic, since the Lagrange multiplier matrix is only being solved to third order in $\Delta t$.

\section{MODEL AND IMPLEMENTATION}

We have applied the algorithms outlined in the previous sections to a system of $N$ dipolar soft spheres (DSS). This system has been the subject of earlier simulations using the quaternion method. ${ }^{16,17}$ The DSS interact with the two-body potential of the form

$$
U=U_{S}+U_{D},
$$

where

$$
U_{S}=4 \epsilon\left(\frac{\sigma}{r_{i j}}\right)^{12}
$$

and

$$
U_{D}=\left(\frac{1}{r_{i j}^{3}}\right)\left(\boldsymbol{\mu}_{i} \cdot \boldsymbol{\mu}_{j}-3\left(\boldsymbol{\mu}_{i} \cdot \hat{\mathbf{r}}_{i j}\right)\left(\boldsymbol{\mu}_{j} \cdot \hat{\mathbf{r}}_{i j}\right)\right),
$$


where $\epsilon$ and $\sigma$ are energy and length scales, $\boldsymbol{\mu}$ are the dipole moments of particles, and $\mathbf{r}_{i j}$ is the separation of particles $i$ and $j$. For a given particle $i$ the dipole moment can be written as

$$
\boldsymbol{\mu}_{i}(t)=\mathscr{Q}_{i}(t) \boldsymbol{\mu}_{\mathrm{ref}},
$$

where we have taken all the reference dipoles parallel to $\hat{\mathbf{z}}$. Both potentials generate a force on the centers of mass, while only the dipolar interaction results in a torque. For the purposes of the present simulations we have truncated the above potentials at a distance $r_{c}=2.5 \sigma$. A shifting function was then added to ensure that both the potentials and the forces approach zero continuously as $r_{c}$ is approached. For the short ranged part of the potential $\left(U_{s}\right)$ we have added a linear term of the form

$$
U_{S} \Rightarrow U_{S}+A r_{i j}+B .
$$

For the dipole-dipole part of the potential we shift only the radial part of the potential, as follows

$$
\frac{1}{r_{i j}^{3}} \Rightarrow \frac{1}{r_{i j}^{3}}+a r_{i j}^{4}+b
$$

The constants $A, B, a$, and $b$ are determined from the continuity condition.

All simulations were carried at a density of $\rho \sigma^{3}=0.5$ with a dipolar strength $\mu^{* 2}=\mu^{2} /\left(\epsilon \sigma^{3}\right)=2$. Initially, all the particles were placed on an fcc lattice. The initial dipole orientations randomly distributed on the unit sphere. To implement the quaternion method, the rotational velocities and their time derivatives, as well as the time derivatives of the quaternions, were taken to be zero initially. In RSHAKE, we took $\Pi_{-1 / 2}=0$ for all the particles. Cubic periodic boundary conditions were used. All particles were assumed to be spherical using a diagonal body-frame moment-ofinertia tensor with $I_{x x}=I_{y y}=I_{z z}=0.025 M \sigma^{2}$, where $M$ is the mass of each particle. For spherical particles, the $J$ matrix [Eq. (20)] reduces to $\frac{1}{2} I$.

\section{RESULTS AND DISCUSSION}

To compare RSHAKE with the standard quaternion method (using a 4th-order Gear predictor/corrector integrator), we have performed simulations on the DSS system using both methods for a variety of system sizes $(N=108,256,500,864)$, and time steps ranging from 0.001 to $0.010 t_{0}$ where $t_{0}=\sqrt{m \sigma^{2} / \epsilon}$. (For SPC water, the time scale $t_{0}$ will be around $2 \mathrm{ps}$.) All reported results are for a fixed total time of $t^{*}=t / t_{0}=252$. All quoted CPU times are for an IBM RS6000 workstation (Model 43P).

In our analysis, we define two measures of energy error: global energy drift, $\epsilon_{g}$, and local energy fluctuations, $\epsilon_{l}$. Both are determined from a plot of the instantaneous total energy (per particle) as a function of time over the entire length of the simulation. Global energy drift is defined as the slope of this curve (determined by a least-squares fit) multiplied by the duration of the simulation. Local fluctuation error is defined as the standard deviation energy about the above linear fit. In Fig. 1 we show energy-versus-time plots

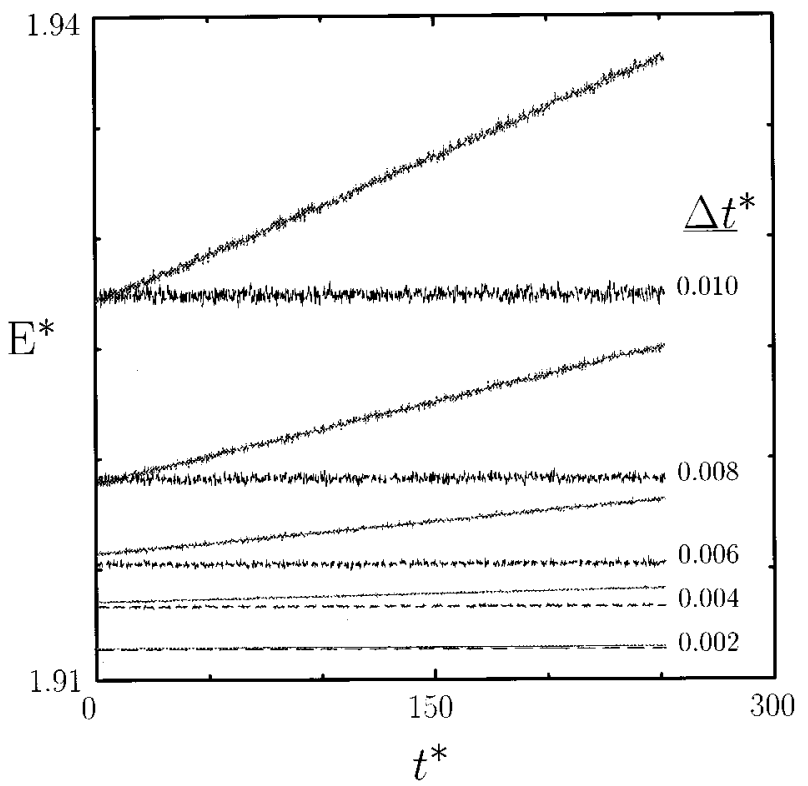

FIG. 1. Total energy as a function of time for a 500 particle DSS system using both RSHAKE (dashed-horizontal lines) and standard quaternions (solid-sloped lines). (The results for a variety of time steps have been shifted relative to each other along the energy axis for clarity.)

for a variety of time steps $\left(\Delta t^{*}=0.002,0.004,0.006,0.008\right.$, $0.010)$ for a DSS system of 500 particles. Results for both quaternions and RSHAKE are shown. (The plots for the different $\Delta t^{*}$ values have been shifted for clarity.) From this figure it is clear that global energy drift for the quaternion method increases rapidly with time step and always dominates the local energy fluctuation for the method. In contrast, the global error for RSHAKE is negligible-being always smaller than the local fluctuations for the method. The local fluctuations in RSHAKE are always larger than those for the quaternion method, due largely to the use of a higher-order (Gear) integrator.

In Table I is shown the derived global energy drift and local energy fluctuations for both methods for two systemsone small $(N=256)$ and one large $(N=864)$ - for a variety of time steps. The CPU time (in seconds) for each run is also reported. Although, for a given time step, the quaternion method is always faster than RSHAKE, the global energy drift of the nonsymplectic quaternion integrator is so large that, for fixed energy error tolerance and simulation length, RSHAKE allows for a much greater time step to be used. To illustrate this, we show in Fig. 2 a $\log -\log$ plot of $\epsilon_{l}$ and $\epsilon_{g}$ for both RSHAKE and our implementation of quaternions. This shows that, for example, for a fixed total error tolerance of 1 part in $10^{-4}$, in our runs a time step of just above $0.002 t_{0}$ would be required for quaternions, whereas $0.007 t_{0}$ would be sufficient for RSHAKE-for larger tolerances the difference is even greater! The slopes of the curves in Fig. 2 give the order of the error measurement for each method. The slopes for the local fluctuations of each method are equal to about 2, indicating an $O\left((\Delta t)^{2}\right)$ method-this makes sense despite the fact that the integrator used for the 
TABLE I. Comparison of RSHAKE with quaternion method.

\begin{tabular}{llccr}
\hline \hline$N$ & $\Delta t^{*}$ & $\epsilon_{l}^{*}$ & $\epsilon_{q}^{*}$ & CPU $(\mathrm{s})$ \\
\hline \multirow{4}{*}{256} & 0.002 & $1.1 \times 10^{-5}$ & $1.2 \times 10^{-6}$ & 5022 \\
& 0.004 & $4.3 \times 10^{-5}$ & $4.7 \times 10^{-6}$ & 2853 \\
& 0.006 & $1.0 \times 10^{-4}$ & $1.5 \times 10^{-5}$ & 2054 \\
& 0.008 & $1.8 \times 10^{-4}$ & $4.8 \times 10^{-5}$ & 1632 \\
& 0.010 & $2.7 \times 10^{-4}$ & $3.0 \times 10^{-5}$ & 1381 \\
864 & 0.002 & $5.8 \times 10^{-6}$ & $6.4 \times 10^{-7}$ & 21391 \\
& 0.004 & $2.3 \times 10^{-5}$ & $6.6 \times 10^{-6}$ & 12167 \\
& 0.006 & $5.2 \times 10^{-5}$ & $8.5 \times 10^{-6}$ & 9421 \\
& 0.008 & $9.5 \times 10^{-5}$ & $1.0 \times 10^{-5}$ & 8069 \\
& 0.010 & $1.5 \times 10^{-4}$ & $1.6 \times 10^{-5}$ & 7619 \\
256 & & $Q^{-5}$ & \\
& 0.002 & $7.4 \times 10^{-6}$ & $6.0 \times 10^{-5}$ & 3306 \\
& 0.004 & $3.0 \times 10^{-5}$ & $5.9 \times 10^{-4}$ & 1762 \\
& 0.006 & $6.9 \times 10^{-5}$ & $2.4 \times 10^{-3}$ & 1246 \\
864 & 0.008 & $1.2 \times 10^{-4}$ & $6.4 \times 10^{-3}$ & 992 \\
& 0.002 & $4.1 \times 10^{-6}$ & $6.1 \times 10^{-5}$ & 15643 \\
& 0.004 & $1.5 \times 10^{-5}$ & $5.8 \times 10^{-4}$ & 9160 \\
& 0.006 & $3.5 \times 10^{-5}$ & $2.4 \times 10^{-3}$ & 7026 \\
& 0.008 & $6.5 \times 10^{-5}$ & $6.4 \times 10^{-3}$ & 5815 \\
\hline \hline
\end{tabular}

quaternions is fourth order, since the integrator for the translational degrees of freedom is the same in both methods and is second order in the time step. The slopes of the global energy-drift curves for RSHAKE and quaternions are about 2 and 3, respectively-the origin of this difference is unclear. To view the same data in a slightly different way for the 500 particle simulations, we plot in Fig. 3 the dominant error for each method (global for quaternions and local for RSHAKE) as a function of CPU time (in seconds) for a fixed length run of $252 t_{0}$ - the different points correspond to different time

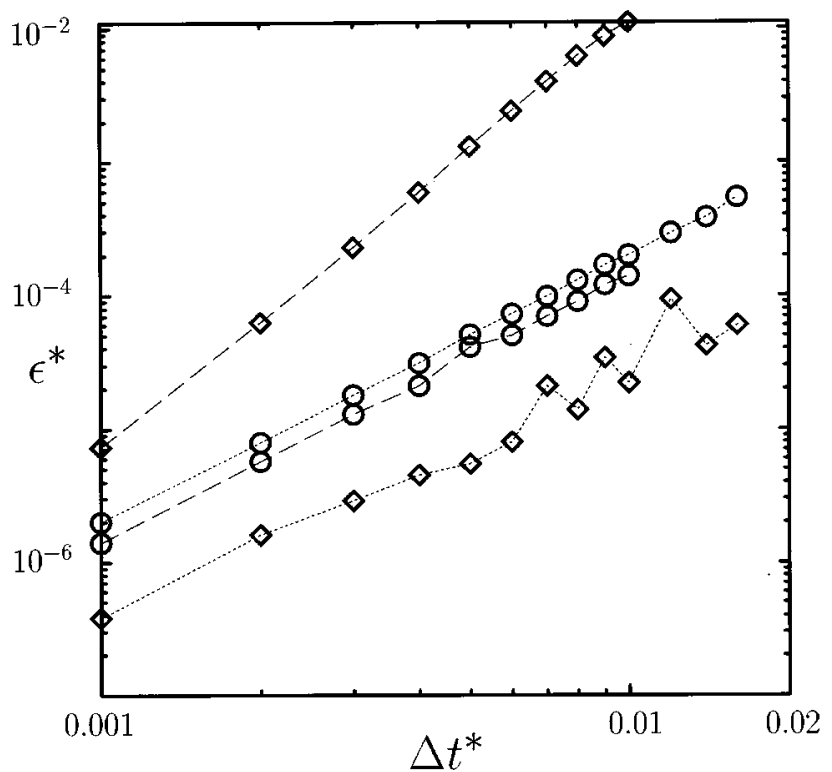

FIG. 2. Global (diamonds) and local (circles) energy errors as functions of time step, $\Delta t$, (as a log-log plot) for a 500 particle DSS system using both RSHAKE (dotted lines) and the 4th-order (Gear) quaternion integrator (dashed lines).

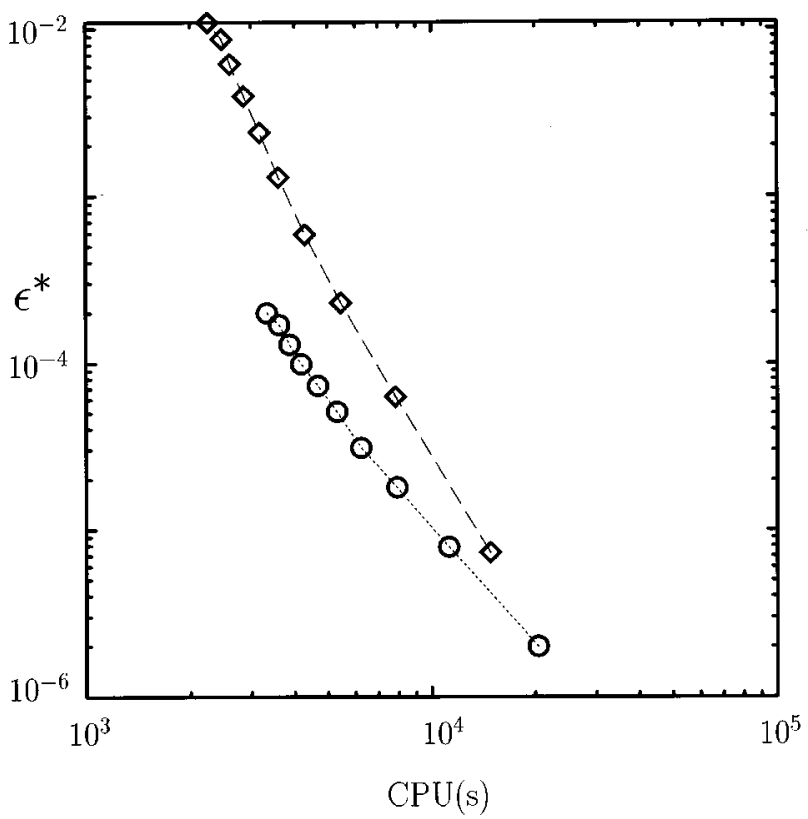

FIG. 3. Dominant energy error as a function of CPU time (in seconds) for a 500 particle DSS system using RSHAKE (circles) and the quaternion integrator (diamonds).

steps. Again, it can be clearly seen that, for fixed energy tolerance, RSHAKE is superior to the standard implementation of quaternions.

To examine the size dependence of our results, we show in Fig. 4 a plot of CPU time versus particle number $N$ for our two methods. The time steps chosen $\left(0.005\right.$ and $0.002 t_{0}$ for RSHAKE and quaternions, respectively) give comparable total energy errors for our $252 t_{0}$ length runs of the 500 particle

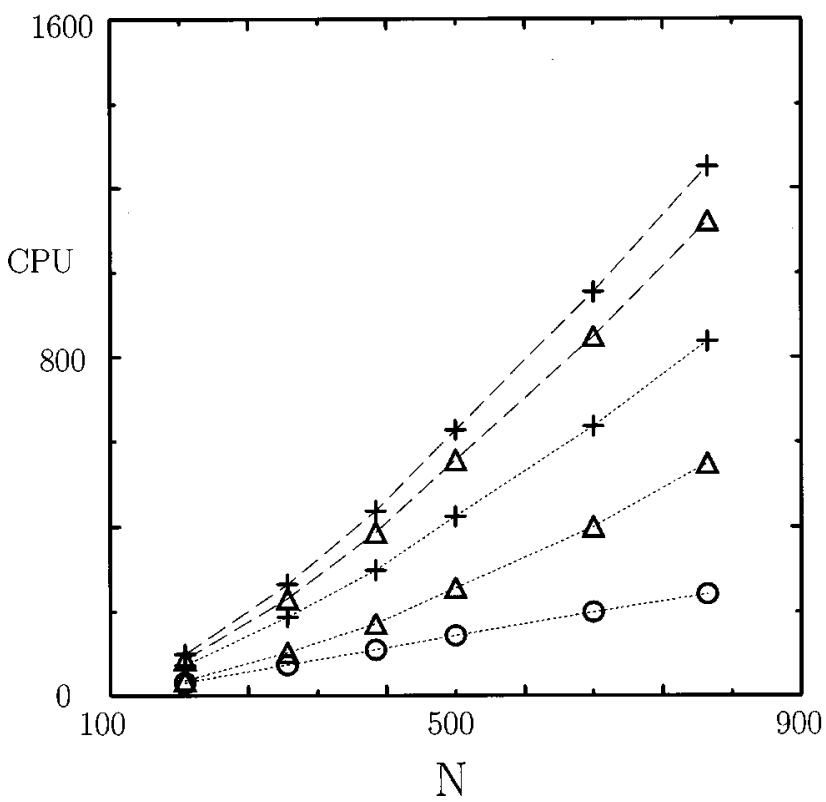

FIG. 4. Total CPU (crosses) as a function of the system size $N$ for RSHAKE (dotted line, $\Delta t=0.005 t_{0}$ ) and quaternions (dashed line, $\Delta t=0.002 t_{0}$ ). Also shown is the CPU time for the force evaluations in both methods (triangles) as well as the CPU time required to solve for the Lagrange multiplier matrix in RSHAKE (circles). 


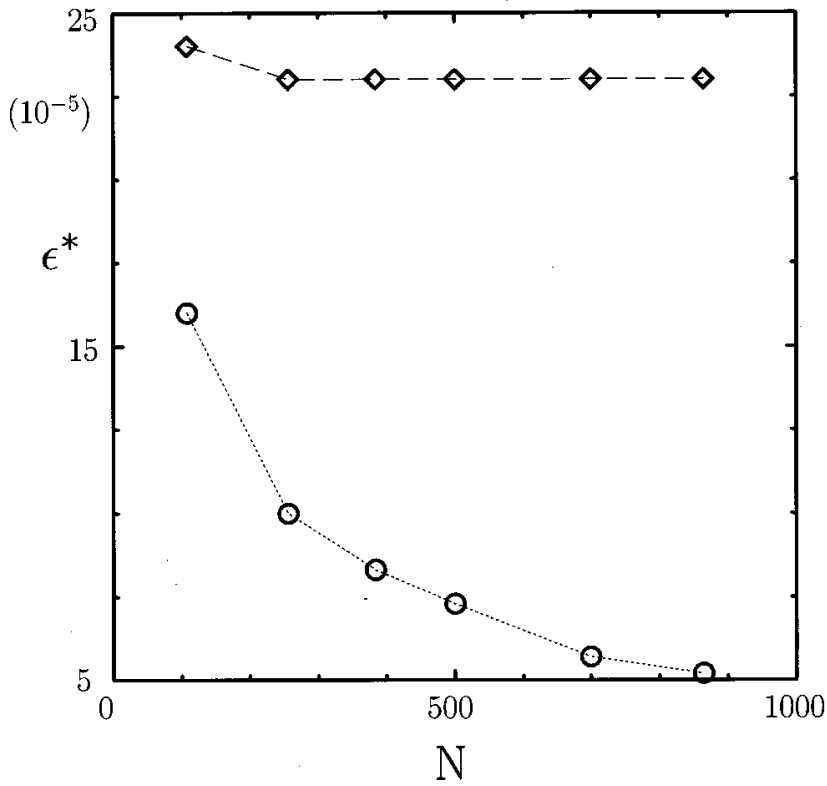

FIG. 5. Dominant energy error as a function of $N$ for RSHAKE (circles, $\Delta t=0.006 t_{0}$, local energy error) and the quaternion integrator (diamonds, $\Delta t=0.003 t_{0}$, global energy error).

system (see Fig. 2). In addition to total CPU time, the amount of time spent in force (and torque) evaluations is plotted for both methods, along with the amount of time required in RSHAKE to solve for the Lagrange multiplier matrix. For all $N$, the RSHAKE method remains the faster algorithm. Also note that, since the Lagrange multiplier determination step scales only linearly with $N$-as opposed to the $N^{2}$ scaling of the force evaluations, this step will become much less important for large system sizes, further increasing the efficiency of RSHAKE. It should also be noted that the local energy fluctuations in the energy per particle will scale as $N^{-1 / 2}$, thereby decreasing with system size, whereas the global energy drift appears to be relatively size independent. Therefore the total error of RSHAKE, being dominated by local fluctuations, will decrease as larger systems are considered, but that for quaternions will remain nearly constant. This is illustrated for our system in Fig. 5, which shows the dominant error for RSHAKE $\left(\Delta t^{*}=0.006\right)$ and quaternions $\left(\Delta t^{*}=0.003\right)$ over our $252 t_{0}$ runs.

We have also studied our system using the implicit method of Fincham for integrating the quaternion equations of motion (Sec. III). Our results show that this method has a significantly larger energy drift for all studied time steps than when the predictor-corrector is used. For example, for a 500 particle system with time step $\Delta t^{*}=0.002$, the global energy error in Fincham's method is $1.1 \times 10^{-3}$ in reduced units, compared to $6.3 \times 10^{-5}$ for predictor-corrector. In a recent paper, ${ }^{18}$ Fincham compared results for a linear molecule using his implicit method and several other methods, including his LEN algorithm, which is shown in Appendix B to be equivalent to RSHAKE applied to a linear molecule. Fincham concluded that LEN (RSHAKE) was superior to his implicit quaternion integrator. Thus, concluding that Fincham's implicit method does not compare well, we have per- formed our detailed comparisons only between quaternions and RSHAKE.

\section{SUMMARY}

We have developed a new algorithm, RSHAKE, for the numerical integration of the equations of motion for the orientational degrees of freedom of rigid molecules. Unlike other standard methods such as quaternions, in which the rotation matrix is parameterized, the entire rotation matrix is treated in RSHAKE as a dynamical variable with corresponding conjugate momentum. The resulting equations of motion for the rotation matrix must then be solved subject to the constraint that the matrix remain orthogonal. In analogy with the standard SHAKE algorithm for molecules with bond constraints, RSHAKE uses a Verlet (leapfrog) integration scheme while simultaneously solving the constraint equations exactly. Like SHAKE and true Hamiltonian dynamics, RSHAKE can be shown to be fully symplectic-a property that gives the method superior stability with respect to energy conservation. For a system of soft-sphere dipoles we have demonstrated that RSHAKE is superior to quaternion-based integration schemes using both a 4th-order (Gear) predictor-corrector method and an implicit leapfroglike algorithm due to Fincham. ${ }^{12}$

\section{ACKNOWLEDGMENTS}

This research was supported by National Science Foundation Grants CHE-950281 (B.B.L and A.K.) and DMS9303223 (B.J.L.) The authors also thank the Kansas Institute for Theoretical and Computational Science and the Department of Applied Mathematics and Theoretical Physics of Cambridge University for providing stimulating research environments during this project.

\section{APPENDIX A: SOLUTION OF THE NONLINEAR EQUATIONS}

We can simplify Eqs. (28)-(29) to

$$
Q_{n+1}=\hat{Q}_{n+1}+2(\Delta t)^{2} Q_{n} \lambda_{n} J^{-1} \text {, }
$$

where $\hat{Q}_{n+1}$ represents the unconstrained step in $Q$ :

$$
\hat{Q}_{n+1}=Q_{n}+\Delta t \Pi_{n-1 / 2} J^{-1}-(\Delta t)^{2} \partial_{Q_{n}} \Phi J^{-1} \text {. }
$$

Substituting Eq. (A1) into Eq. (30) and multiplying on either side by $J$ results in a quadratic (matrix Riccati) equation for the six independent elements of the (symmetric) $\lambda$ matrix:

$$
F\left(\lambda_{n}\right)=\alpha \lambda_{n}^{2}+\lambda_{n} B+B^{T} \lambda_{n}+C=0,
$$

where

$$
\begin{aligned}
& \alpha=2(\Delta t)^{2}, \quad B=Q_{n}^{T} \hat{Q}_{n+1} J, \\
& C=\frac{1}{2(\Delta t)^{2}} J\left(\hat{Q}_{n+1}^{T} \hat{Q}_{n+1}-I\right) J .
\end{aligned}
$$

Observe that from Eq. (A2), and the fact that $\lambda$ is bounded as $\Delta t \rightarrow 0$, the matrix $C$ also remains bounded as $\Delta t \rightarrow 0$. 
Following the standard approach, we localize the solution of this nonlinear equation by assuming that we have some initial guess $\bar{\lambda}$ (such as $\lambda_{n-1}$ ) near to the solution $\lambda_{n}$. We compute an improved approximation by linearizing the equation

$$
F(\bar{\lambda}+\Delta)=0,
$$

and solving for $\Delta$. This gives $\Delta$ as the solution of the matrix Lyapunov equation

$$
K^{T} \Delta+\Delta K=-F(\bar{\lambda}), \quad K=\alpha \bar{\lambda}+B .
$$

We then obtain a corrected value

$$
\hat{\lambda}=\bar{\lambda}+\Delta \text {. }
$$

The iteration of this process is just the Newton method, which, started from an initial guess $\lambda^{(0)}$ computes a sequence of approximations $\lambda^{(1)}, \lambda^{(2)}, \ldots$ to $\lambda_{n}$ recursively from

$$
\lambda^{(m)}=\lambda^{(m-1)}+\Delta^{(m)},
$$

where

$$
K_{m}^{T} \Delta^{(m)}+\Delta^{(m)} K_{m}=-F\left(\lambda^{(m-1)}\right), \quad K_{m}=\alpha \lambda^{(m-1)}+B .
$$

Although there is a substantial body of literature on the efficient solution of matrix Riccati and Lyapunov equations, this research is primarily oriented to the treatment of largedimensional problems. In our case, we need to solve many small-dimensional decoupled problems. For such smalldimensional problems, the straightforward methods are probably optimal. The simplest approach is to write Eq. (A5) as a six-dimensional vector equation, requiring the factorization of a $6 \times 6$ matrix at each Newton iteration step.

There are several possible ways to reduce the complexity of the Newton step, all obtained by different simplifications of the Lyapunov equation (A5):

Observe that $K$ is an $O(\Delta t)$ perturbation of $J$, and replace $K$ by $J$ in Eq. (A5). This results in a modified Newton iteration which is linearly, not quadratically, convergent, with rate of convergence $\rho=O(\Delta t)$. Write Eq. (A5) for the components of $\Delta^{(m)}$ in standard linear algebraic form as a system,

$W_{m} \hat{\Delta}^{(m)}=b$,

where $\hat{\Delta}^{(m)} \in \mathbf{R}^{6}$ is a vector composed of the entries in the upper triangular part of $\Delta, b$ is the corresponding part of the right hand side of Eq. (A5), and $W_{m}$ is a $6 \times 6$ matrix determined from the components of $K$. Instead of inverting (i.e., factoring) $W_{m}$ at each iteration, compute and factor $W$ once, at the beginning of the time step, and use these factors to solve the problem at each step of the iteration. This method is also only linearly convergent, with rate $\rho=O(\Delta t)$, but we expect this to be faster than variant (i).

(iii) In case $J=\beta 1$, with scalar $\beta$, observe that the matrix $B$ is also nearly a scalar multiple of the identity and that $K_{m}$ and $\Delta^{(m)}$ will commute to second order,
$\Delta^{(m)} K_{m}=K_{m} \Delta^{(m)}+O\left(\Delta t^{2}\right)$.

Replacing $\Delta^{(m)} K_{m}$ by $K_{m} \Delta^{(m)}$ we obtain an equation

$\left(K_{m}^{T}+K_{m}\right) \Delta^{(m)}=-F\left(\lambda_{n}^{(m-1)}\right)$.

This equation generally does not have a solution with symmetric $\Delta^{(m)}$, but we enforce the symmetry directly by solving for only the upper triangular part of $\Delta^{(m)}$ and then reflecting this about the diagonal to obtain the update. The latter operation can be viewed as a projection from the class of $3 \times 3$ matrices onto the symmetric matrices. The combination of these operations can be viewed as equivalent to an $O\left(\Delta t^{2}\right)$ alteration of the matrix $W_{m}$ in the Newton iteration, hence this scheme would be expected to yield an algorithm that gains two orders of the step size at each iteration.

The modified iterations will typically require several more iterations per time step than the Newton iteration to achieve the same tolerance, although they have the advantage of reduced complexity. In our implementation the iteration is considered converged if the norm of $\Delta$ is less than $10^{-15}$ or Eq. (A4) is satisfied within $10^{-20}$. We set the maximum number of iterations to 50 , which was necessary for the largest time steps that we have studied.

In our examples, $J$ was a scalar multiple of the identity. Initially we took the simplest initial guess for the Lagrange multipliers: the value from the previous step. Even with such a simple minded guess, variant (iii) of the Newton method performed better than the standard Newton. For the softsphere dipole system we consider in Sec. V, this is faster than standard Newton by $60 \%$ for $\Delta t=0.005$. One can improve the speed by improving the initial guess, for example, by keeping the previous three Lagrange multipliers and extrapolating from these points. For large time steps this might be beneficial, although it did not have much of an effect in our simulations. Also note that, as the number of particles is increased, the relative time spent in solving $\lambda$ gets smaller because the total time consumed goes linearly with number of particles-compared to the time required to evaluate forces and torques, which increases superlinearly with particle number (in general quadratically if no cutoffs or special force approximations are used).

Variant (i) of the modified Newton iteration was not competitive with either (iii) or the standard Newton. We did not implement (ii).

\section{APPENDIX B: RSHAKE FOR LINEAR AND PLANAR MOLECULES}

For a linear molecule, assumed to be aligned in the reference $x$ direction, only the $J_{11}$ element of the $J$ matrix is nonzero. Therefore $J$ is not invertible, preventing the straightforward implementation of Eqs. (25)-(27), and modifications must be made. As before, the Lagrangian for a single rigid body is 


$$
\begin{aligned}
\mathscr{C}= & \frac{M}{2} \dot{\mathbf{r}}_{\text {c.m. }} \dot{\mathbf{r}}_{\mathrm{c} . \mathrm{m} .}^{T}+\frac{1}{2} \operatorname{Tr}\left[\dot{Q} J \dot{\mathscr{Q}}^{T}\right]-\Phi\left(\mathcal{Q}, \mathbf{r}_{\text {c.m. }}\right) \\
& +\operatorname{Tr}\left[\lambda\left(\mathscr{Q}^{T} \mathscr{Q}-1\right)\right] .
\end{aligned}
$$

In the linear molecule case, the trace operation in the rotational kinetic energy term (second term) involves only $\dot{\mathscr{Q}}_{\alpha 1}$, where $\alpha=1,2,3$. Also, the potential energy depends only on $Q_{\alpha 1}$, because the position of any atom in the laboratory frame will be the rotation of a reference coordinate that lies on the $x$ axis of the body frame; that is, the position of the $i$ th atom is given by

$$
r_{\alpha}^{i}(t)=Q_{\alpha 1}(t) R_{1}^{i} .
$$

As a result, the potential energy depends only on $\mathscr{Q}_{\alpha 1}$ and the center-of-mass coordinate $\mathbf{r}_{\text {c.m. }}$. The behavior of $\mathcal{Q}_{\alpha \beta}$ for $\beta=2,3$ is irrelevant to the dynamics of the system. In other words, a description of the orientation of a linear molecule requires only the specification of a single vector, which in this case is the first column of the rotation matrix. Thus, the only relevant constraint is that this vector has unit magnitude

$$
Q_{\alpha 1} \mathscr{Q}_{\alpha 1}-1=0 \text {. }
$$

In summary, the Lagrangian and the corresponding rotational equations of motion for a linear molecule are

$$
\begin{aligned}
\mathscr{C}= & \frac{M}{2} \dot{\mathbf{r}}_{\text {c.m. }} \cdot \dot{\mathbf{r}}_{\text {c.m. }}+\frac{1}{2}\left[\dot{\mathscr{Q}}_{\alpha 1} J_{11} \dot{\mathscr{Q}}_{\alpha 1}\right]-\Phi\left(\mathscr{Q}_{\alpha 1}, \mathbf{r}_{\mathrm{c} . \mathrm{m} .}\right) \\
& +\lambda_{11}\left(\mathscr{Q}_{1 \alpha}^{T} \mathscr{Q}_{\alpha 1}-1\right),
\end{aligned}
$$

and

$$
\begin{aligned}
& \Pi_{\alpha 1}=\dot{\mathscr{Q}}_{\alpha 1} J_{11}, \\
& \dot{\Pi}_{\alpha 1}=-\partial_{\ell_{\alpha 1}} \Phi+2 \varrho_{\alpha 1} \lambda_{11} \text {, } \\
& g(Q)=Q_{1 \alpha}^{T} Q_{\alpha 1}-1=0,
\end{aligned}
$$

with the RSHAKE algorithm becomes

$$
\begin{aligned}
& \Pi_{\alpha 1}^{n+1 / 2}=\Pi_{\alpha 1}^{n-1 / 2}+\Delta t\left[2 \mathscr{Q}_{\alpha 1}^{n} \lambda_{11}^{n}-\partial_{\mathscr{Q}_{\alpha 1}^{n}} \Phi\right], \\
& \mathscr{Q}_{\alpha 1}^{n+1}=\mathscr{Q}_{\alpha 1}^{n}+\Delta t \Pi_{\alpha 1}^{n+1 / 2} / J_{11}, \\
& g\left(\mathscr{Q}^{n+1}\right)=0 .
\end{aligned}
$$

In this case the constraint Eq. (B10) is simply a quadratic equation for $\lambda_{11}^{n}$ which can be solved exactly. The above algorithm for a linear molecule has appeared in the literature $^{18}$ and is referred to as the LEN method. Fincham showed that LEN is remarkably stable and superior to his implicit, explicit, and ORT methods.

For a planar molecule, assumed to lie entirely in the $x-y$ plane of the body frame, the $J$ matrix will have $J_{11}$ and $J_{22}$ as the only nonzero elements. In analogy with the linear case, the potential energy will only depend upon $Q_{\alpha 1}$ and $Q_{\alpha 2}$
TABLE II. Dimensions of parameters for different molecular geometries.

\begin{tabular}{ccccc}
\hline \hline Molecule & $Q$ & $\Pi$ & $J$ & $\lambda$ \\
\hline 3D & $3 \times 3$ & $3 \times 3$ & $3 \times 3$ & $3 \times 3$ \\
Planar & $3 \times 2$ & $3 \times 2$ & $2 \times 2$ & $2 \times 2$ \\
Linear & $3 \times 1$ & $3 \times 1$ & $1 \times 1$ & $1 \times 1$ \\
\hline \hline
\end{tabular}

together with $\mathbf{r}_{\text {c.m. }}$. In this case the evolution of $\mathcal{Q}_{\alpha 3}$ is irrelevant-only two noncolinear vectors are necessary to describe a planar molecule. In this representation these two vectors are the first and second columns of the rotation matrix. The rotational equations of motion for a planar molecule are then

$$
\begin{aligned}
& \Pi_{\alpha i}=\dot{\mathscr{Q}}_{\alpha j} J_{j i}, \\
& \dot{\Pi}_{\alpha i}=-\partial_{Q_{\alpha i}} \Phi+2 Q_{\alpha j} \lambda_{j i}, \\
& g(\varrho)=\mathscr{Q}_{i \alpha}^{T} Q_{\alpha j}-\delta_{i j}=0,
\end{aligned}
$$

where $\alpha=1,2,3$ and $i, j=1,2$ and repeated indices are summed over. The RSHAKE algorithm for a planar molecule reduces to

$$
\begin{aligned}
& \Pi_{\alpha i}^{n+1 / 2}=\Pi_{\alpha i}^{n-1 / 2}+\Delta t\left[2 \mathscr{Q}_{\alpha j}^{n} \lambda_{j i}^{n}-\partial_{Q_{\alpha i}^{n}} \Phi\right], \\
& \mathscr{C}_{\alpha i}^{n+1}=\mathscr{Q}_{\alpha i}^{n}+\Delta t \Pi_{\alpha j}^{n+1 / 2}\left(J^{-1}\right)_{j i}, \\
& g\left(\mathscr{Q}^{n+1}\right)=0 .
\end{aligned}
$$

In summary, whether the molecule is linear, planar, or three dimensional, the equations of motion and the corresponding leapfrog algorithm are described by Eqs. (25)-(27) and Eqs. (29) and (30) with the understanding that the dimensions of the variables are given as in Table II.

${ }^{1}$ H. Goldstein, Classical Mechanics (Addison-Wesley, Reading, MA, 1980).

${ }^{2}$ D. J. Evans, Mol. Phys. 34, 317 (1977).

${ }^{3}$ M. A. Allen and D. J. Tildesley, Computer Simulation of Liquids (Oxford Science, Oxford, 1987).

${ }^{4}$ J. M. Sanz-Serna and M. P. Calvo, Numerical Hamiltonian Problems (Chapman and Hall, New York, 1995).

${ }^{5}$ V. I. Arnold, Mathematical Methods of Classical Mechanics (SpringerVerlag, New York, 1978).

${ }^{6}$ J. Frank, W. Huang, and B. Leimkuhler, J. Comput. Phys. (in press).

${ }^{7}$ J. P. Ryckaert, G. Ciccotti, and H. J. C. Berendsen, J. Comput. Phys. 23, 327 (1977).

${ }^{8}$ H. C. Andersen, J. Comput. Phys. 52, 24 (1983).

${ }^{9}$ B. Leimkuhler and R. D. Skeel, J. Comput. Phys. 112, 117 (1994).

${ }^{10}$ R. I. McLachlan and C. Scovel, J. Nonlinear Sci. 5, 233 (1995).

${ }^{11}$ S. Reich, Physica D 76, 375-383 (1994).

${ }^{12}$ D. Fincham, Mol. Simul. 8, 165 (1992).

${ }^{13}$ B. Leimkuhler and S. Reich, Math. Comp. 63, 589 (1994).

${ }^{14}$ D. J. Dichmann and J. H. Maddocks, J. Nonlinear Sci. 6, 271 (1992).

${ }^{15}$ R. Ahlrichs and S. Brode, Comput. Phys. Commun. 42, 59 (1986).

${ }^{16}$ P. G. Kusalik, J. Chem. Phys. 93, 3520 (1990).

${ }^{17}$ D. Wei and G. N. Patey, Phys. Rev. A 46, 2043 (1992).

${ }^{18}$ D. Fincham, Mol. Simul. 11, 79 (1993). 